\title{
Unique management considerations for rectal cancer and colon cancer
}

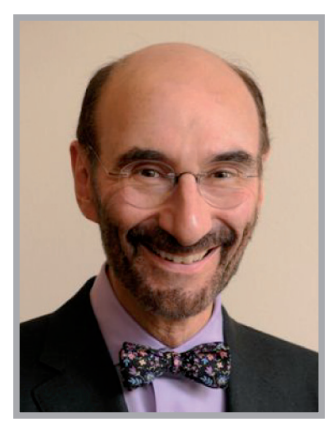

Bruce D Minsky*: Bruce D Minsky recently joined the Department of Radiation Oncology at the MD Anderson Cancer Center in Houston (TX, USA) as a Professor and Director of Clinical Research. Dr Minsky received his medical degree from the University of Massachusetts (USA). He completed an internship at New England Deaconess Hospital (MA, USA), and a residency in radiation therapy at Harvard Joint Center for Radiation Therapy (MA, USA). He spent 20 years in New York at the Memorial Sloan Kettering Cancer Center and was the Vice Chairman of Radiation Oncology and a Professor of Radiation Oncology in Medicine at Cornell University. From 2007 to 2012 he was a Professor of Radiation and Cellular Oncology and Associate Dean in the Biological Sciences Division at the University of Chicago (IL, USA). In 2004, Dr Minsky received a medical degree honoris causa from Friedrich Alexander University, Erlangen, Germany in recognition for his work in gastrointestinal cancers. Dr Minsky is an editorial board member of several journals and has served on the board of directors of the American Society of Clinical Oncology and the American Society for Therapeutic Radiology and Oncology Board of Directors.

\section{Q How does incidence of local} recurrence vary with colon cancer and rectal cancer?

The incidence of local recurrence of rectal cancer is much higher than colon cancer. The reason for this is the surgical challenge of operating in the pelvis as compared with the abdomen.

Q In light of this, how does the management of radiation differ for patients with colon cancer and rectal cancer?

Radiation, in most cases, is not used in patients with colon cancer. There are two types of preoperative radiation treatments for rectal cancer; short-course radiation and long-course radiation plus concurrent chemotherapy (chemoradiation). There are randomized trials comparing the two.

The rationale for radiation for rectal cancer is to address the higher incidence of local recurrence. Pelvic radiation, whether it is short-course or long-course chemoradiation, is limited to patients with rectal cancer (tumors within the pelvis).

Q In what cases would you use radiation treatment in colon cancer?

The use of radiation in colon cancer is limited to cT4 tumors in the pelvis. There are clinical settings where a low sigmoid tumor or cecal cancer are adherent to an organ or structure within the
News \& Views

News

Journal Watch

Ask the Experts

Interview 
pelvis, such as the bladder or the pelvic side wall. In these cases, the risk of local recurrence is high enough to justify the use of radiation. The patient would be treated in a similar manner as with rectal cancer (chemoradiation).

\section{Q There has been some controversy} as to whether all node-positive rectal cancer patients require postoperative chemotherapy. What is your view?

The standard of care for cT3-4 and/or node positive rectal cancer is preoperative chemoradiation followed by surgery and 4 months of postoperative adjuvant chemotherapy. Two randomized European trials published within the past few years have challenged that approach, suggesting that postoperative chemotherapy may not be necessary for patients who receive preoperative chemoradiation for rectal cancer (EORTC 22921 and FFCD 9203). There is significant controversy surrounding those trials due their overall design, and the number of patients who declined postoperative chemotherapy. In my view, given multiple positive randomized trials in the adjuvant colon setting, the standard of care remains 4 months of postoperative chemotherapy. However, there are investigators in some of the European countries who feel that it is not necessary based on those two randomized trials.

Q What were the controversies of these two randomized trials?

As discussed above, the two randomized trials showed no benefit in overall survival when chemotherapy was added in the postoperative setting. However, a large body of data, both in adjuvant colon and rectal cancer, suggests that a total of 6 months adjuvant chemotherapy significantly increases survival. Therefore, although those two studies were negative, most oncologists still advocate the use of postoperative chemotherapy following preoperative chemoradiation.

Q How does the response rate affect the type of surgery used following chemoradiation?

The higher the rectal tumor response rate, the greater the likelihood that we can use sphincter-sparing surgery. For example, patients who initially require an abdominal perineal resection may be able to undergo a sphincter-sparing coloanal anastomosis if there is a favorable tumor response. However, the ability to perform this is dependent on a number of variables such as the response rate, proximity of the tumor to the dentate line, gender, body habitus, and the skill and commitment of the surgeon.

\section{Q Are there any other unique} management considerations for patients with rectal cancer compared with those with colon cancer?

With colon cancer, sphincter preservation is not an issue because the tumor is above the pelvis. The unique surgical considerations for rectal cancer are related to the challenge of operating on the pelvis: trying to maintain both urinary, sphincter and sexual function for the patient, while completely removing the tumor. Local control and preservation of function are the unique end points in rectal cancer. In the colon, preservation of pelvic organ function is not an issue.

\section{Q How do you feel that the management of rectal cancer can improve?}

There will be further refinement of preoperative therapies. For example, some patients could be treated with more conservative chemotherapy, radiation and surgery. I think that some more conservative surgical approaches, such as local excision, show promise. Additional avenues of research include the development of new chemotherapeutic agents combined with radiation, and identifying subsets of patients who may not need pelvic radiation.

Q How positive are you about the way that research is progressing?

It is a slow but steady process. I believe that the new series of questions being asked, which include not just developing innovative therapies but also challenging us to identify more conservative or selective approaches, provide a robust clinical research portfolio. 
Financial \& competing interests disclosure $B D$ Minsky has no relevant affiliations or financial involvement with any organization or entity with a financial interest in or financial conflict with the subject matter or materials discussed in the manuscript. This includes employment, consultancies, honoraria, stock ownership or options, expert testimony, grants or patents received or pending, or royalties.

No writing assistance was utilized in the production of this manuscript.

The editorial team is eager to receive any comments our readers might have on this topic for potential publication in future issues. Please direct any such communications to:

Ruth Williamson, Commissioning Editor, Colorectal Cancer

ruth.williamson@futuremedicine.com 